Supporting Information

\title{
Enhanced Cavitation and Hydration Crossover of Stretched Water in the Presence of $\mathrm{C}_{60}$
}

\author{
Sa Hoon Min, * Sidath Wijesinghe and Max L. Berkowitz* \\ Department of Chemistry, The University of North Carolina at Chapel Hill, Chapel Hill, \\ North Carolina 27599, United States
}

*E-mail: sahoon@email.unc.edu,maxb@unc.edu 


\section{SIMULATION DETAILS}

Methods: Molecular dynamics (MD) simulations were performed to study a spontaneous cavitation of water in the presence of $\mathrm{C}_{60}$ impurity. A simulation box of $4.5^{3} \mathrm{~nm}^{3}$ in size was used for simulations of pure water and also water with single $\mathrm{C}_{60}$ molecule. For the mean-first passage time (MFPT) analysis, 200 independent initial conformations of each system were equilibrated by 20 -ns isothermal-isobaric (NPT) MD simulations at $T=300 \mathrm{~K}$ and ambient pressure. The spontaneous cavitation was induced in NPT MD simulations at $p=-185 \mathrm{MPa}$. A time step of 2 fs was employed, and the coordinates were saved every 100 fs. Periodic boundary conditions for all directions were applied. A cutoff for the short-range nonbonded interactions was $1.0 \mathrm{~nm}$, and the particle mesh Ewald ${ }^{1}$ was applied for the long-range electrostatic interactions. The bond length of water molecules and $\mathrm{C}_{60}$ buckyballs were constrained using the SETTLE $^{2}$ and LINCS ${ }^{3}$ algorithms, respectively.

Hydrogen Bond Analysis: Hydrogen bond (HB) between water pairs was identified by using a geometric criteria; the distance between donor and acceptor oxygen and the distance between donor hydrogen and acceptor oxygen were less than 0.35 and $0.25 \mathrm{~nm}$, respectively, and simultaneously the acceptor-donor-hydrogen angle was less than $30^{\circ} .^{4}$ The same trajectories used in the analysis of water reorientation were adopted to analyze the HB lifetime.

A time correlation function for the intermittent HB is defined as follows, ${ }^{5}$

$$
C_{\mathrm{HB}}(t)=\frac{\langle h(0) h(t)\rangle}{\langle h(0)\rangle}
$$

where $h(t)=1$ for a water pair forming HB at time $t$ and 0 otherwise. The intermittent HB lifetime was calculated by integrating the time correlation function:

$$
\tau_{\mathrm{int}}=\int_{0}^{\infty} C_{\mathrm{HB}}(t) d t
$$



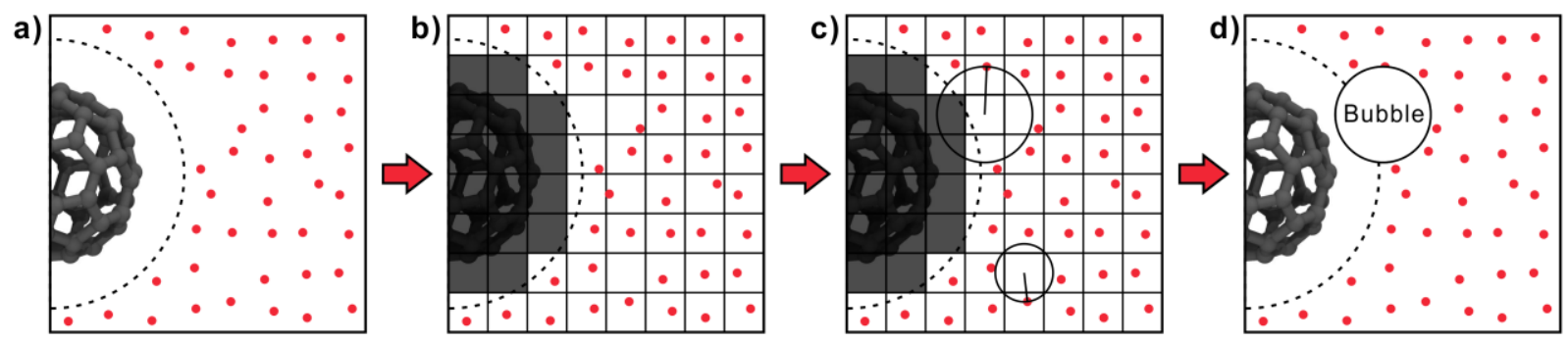

Figure S1. Schematic illustration of the modified largest spherical cavity (LSC) method for finding a bubble in the presence of $\mathrm{C}_{60}$ impurity. (a) Atomic coordinates of water oxygen and $\mathrm{C}_{60}$. The red dots represent water oxygens and the dotted black line represent a sphere where the first hydration shell begins. (b) Gridding with $\sim 0.1^{3} \mathrm{~nm}^{3}$ cubes and (c) finding the LSC based on the center of cubes except the ones located inside the sphere (gray cubes). (d) Bubble detected using the modified LSC method.

The largest spherical cavity (LSC) ${ }^{6}$ method was modified for detecting a bubble in the presence of $\mathrm{C}_{60}$. Firstly, a simulation box was divided into small cubes whose side was about 0.1 $\mathrm{nm}$. For every cube the shortest distance to the position of water oxygen was calculated, and the radius of the LSC was determined from the largest distance among them. If the center of cubes was inside a sphere with a radius of $0.6 \mathrm{~nm}$ from the center position of $\mathrm{C}_{60}$, the cubes were excluded from finding the LSC. Because the LSC method overestimates the volume of very small bubbles, the center position of bubbles was traced only if the volume was greater than $0.285 \mathrm{~nm}^{3}$, which is the largest bubble volume of pure water at ambient condition. We neglected the overlap between the sphere around $\mathrm{C}_{60}$ and the LSC. The center position of the bubble was also measured simultaneously. 


\section{DETAILED INFORMATION ON THE SIMUATION DATA}

Table S1. Summary of the MFPT analysis.

\begin{tabular}{cccc}
\hline System & $\tau_{\mathrm{J}}(\mathrm{ns})^{a}$ & $J\left(10^{26} \mathrm{~cm}^{-3} \mathrm{~s}^{-1}\right)^{b}$ & $V_{\mathrm{c}}\left(\mathrm{nm}^{3}\right)^{c}$ \\
\hline Pure water & 25.6 & 3.84 & 0.851 \\
Single $\mathrm{C}_{60}$ solution & 16.5 & 5.96 & 0.851
\end{tabular}

${ }^{a}$ Nucleation time. ${ }^{b}$ Nucleation rate. ${ }^{c}$ Critical volume.

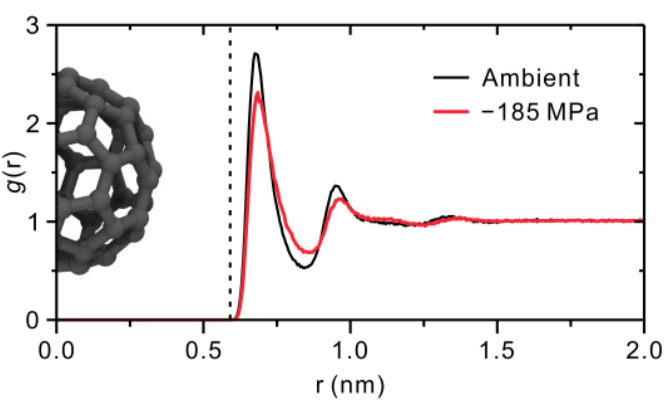

Figure S2. Radial distribution function of water oxygen with respect to the center of $\mathrm{C}_{60}$ at ambient pressure and at $p=-185 \mathrm{MPa}(T=300 \mathrm{~K})$. Water oxygen atoms first appear at $0.6 \mathrm{~nm}$ (black dotted line). 

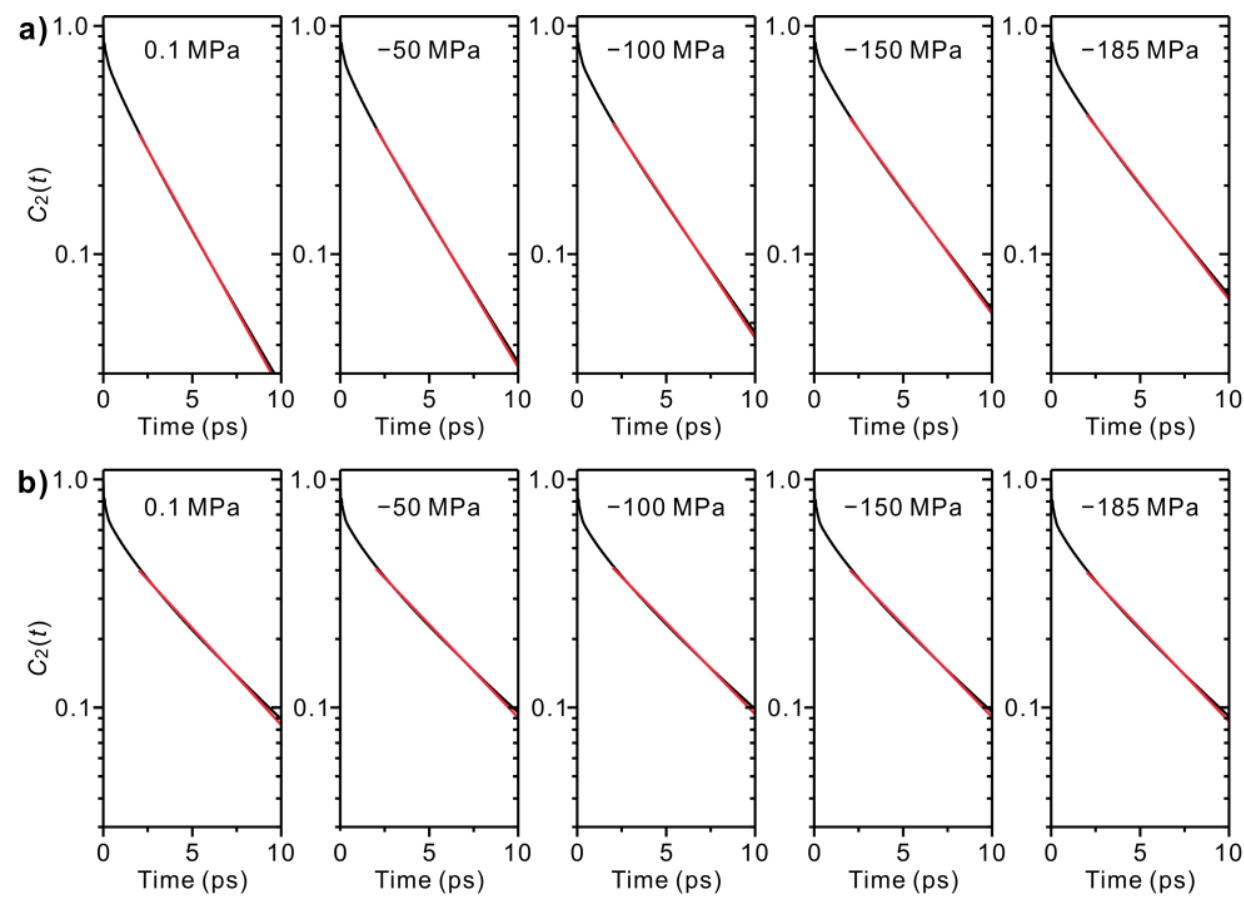

Figure S3. Second-rank orientation time correlation function $C_{2}(t)$ for the $\mathrm{OH}$ vector of (a) pure water and (b) water molecules in the first hydration shell of $\mathrm{C}_{60}$ at different negative pressures. The red line represents an exponential fit in the range of $2-10 \mathrm{ps}$.

\section{INFLUECE OF FORCE FIELD ON CROSSOVER EFFECT}

To find out if the crossover in hydrophobic behavior is not an artifact that depends on specifically chosen force field, we performed extra sets of simulations with three other force fields and calculated intermittent HB lifetimes in these simulations. In one of the force fields to describe $\mathrm{C}_{60}$, we used AMBER03 force field ${ }^{7}$ which has been applied to MD simulations for carbon nanotubes. ${ }^{8}$ In another set of simulations, we used popular OPLS-AA parameters for $\mathrm{C}_{60},{ }^{9}$ and finally in the third set of simulations we used Lennard-Jones parameters for $\mathrm{C}_{60}$ taken from the work of Werder et al. ${ }^{10}$ In all these simulations water was described by TIP4P/2005, and standard combination rules for description of oxygen-carbon interactions were applied. The results for the intermittent HB lifetimes are shown in the figure S4 below. 


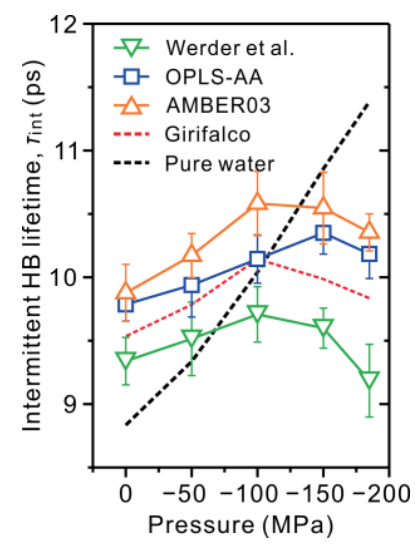

Figure S4. Intermittent hydrogen bond (HB) lifetime $\tau_{\text {int }}$ of water molecules in the first hydration shell of $\mathrm{C}_{60}$ using different force fields for $\mathrm{C}_{60}$ buckyball: AMBER03, OPLS-AA, and the Lennard-Jones parameters from the work of Werder et al. Red and black dashed lines are the same as in Figure $2 b$ of the main text.

As shown in Figure S4, the crossover effect of hydrophobicity was also observed when using other force fields. The simulations clearly show that for all force fields we used, the enhanced hydrogen bonding network of water molecules in the first hydration shell cannot be maintained at high degrees of negative pressure. Interestingly, for 3 out of 4 force fields the crossover occurs at pressure below -100 MPa. 


\section{REFERENCES}

(1) Essmann, U.; Perera, L.; Berkowitz, M. L.; Darden, T.; Lee, H.; Pedersen, L. G. J. Chem. Phys. 1995, 103, 8577-8593.

(2) Miyamoto, S.; Kollman, P. A. J. Comput. Chem. 1992, 13, 952-962.

(3) Hess, B.; Bekker, H.; Berendsen, H. J. C.; Fraaije, J. G. E. M. J. Comput. Chem. 1997, 18, $1463-1472$.

(4) Laage, D.; Hynes, J. T. J. Phys. Chem. B 2008, 112, 14230-14242.

(5) Luzar, A.; Chandler, D. Nature 1996, 379, 55-57.

(6) Gonzalez, M. A.; Abascal, J. L. F.; Valeriani, C.; Bresme, F. J. Chem. Phys. 2015, 142, 154903.

(7) Duan, Y.; Wu, C.; Chowdhury, S.; Lee, M. C.; Xiong, G.; Zhang, W.; Yang, R.; Cieplak, P.; Luo, R.; Lee, T.; Caldwell, J.; Wang, J.; Kollman, P. J. Comput. Chem. 2003, 24, 1999-2012.

(8) Liu, L.; Patey, G. N. J. Chem. Phys. 2014, 141, 18 C518.

(9) Jorgensen, W. L.; Maxwell, D. S.; Tirado-Rives, J. J. Am. Chem. Soc. 1996, 118, 1122511236.

(10) Werder, T.; Walther, J. H.; Jaffe, R. L.; Halicioglu, T.; Koumoutsakos, P. J. Phys. Chem. B 2003, 107, 1345-1352. 